\title{
Dual-stent retrieval for mechanical thrombectomy of refractory clot in acute stroke as a rescue technique
}

\author{
Satya Narayana Patro MD, Daniela Iancu MD MSc
}

Cite as: CMAJ 2017 May 1;189:E634-7. doi: 10.1503/cmaj.160472

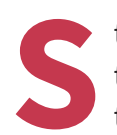

troke is a major public health problem worldwide and is the third most expensive health condition in developed countries. More than $80 \%$ of strokes are thromboembolic ischemic, with the rest hemorrhagic. About $20 \%$ to $30 \%$ of all acute ischemic strokes are caused by large intracranial vessel occlusion, and timely revascularization is associated with significantly improved clinical outcomes and reduced mortality. ${ }^{1}$ The success of intravenous tissue plasminogen activator is limited in acute large-vessel occlusion, with only $7 \%$ to $12 \%$ of cases achieving excellent clinical outcomes. ${ }^{2}$ Recent landmark randomized controlled trials have shown that mechanical thrombectomy using a stent retriever is a fast and effective treatment for patients with occlusive acute largevessel ischemic stroke. ${ }^{3-7}$ However, even with the high success rates seen after standard mechanical thrombectomy using stent retrieval, nearly one-third of patients with occlusive large-vessel ischemic stroke are left without adequate recanalization. ${ }^{8}$

Variable success rates have been achieved with different rescue methods, such as intra-arterial tissue plasminogen activator, aspiration thrombectomy, mechanical thrombus disruption and balloon angioplasty with or without stent placement. Here, we describe an off-label application of dual stent retrievers for mechanical thrombectomy of proximal large-vessel occlusion after failed thrombectomy using a single stent retriever. The dual stent retriever technique may be particularly helpful for refractory clots involving arterial bifurcation, which are resistant to multiple passes of a single stent retriever during mechanical thrombectomy. With the dual technique, there is a greater possibility of retrieving clots, because it allows more coverage of the thrombus within the stent struts and may lead to better outcomes.

\section{What is mechanical thrombectomy using a dual stent retriever?}

Mechanical thrombectomy using a dual stent retriever is a novel technique that involves simultaneous deployment of two stent retrievers side by side into the clot at the arterial bifurcation and retrieval as a single unit for thrombectomy.

Stent retriever technology is the current standard for mechanical thrombectomy in acute ischemic stroke by retrieval of a self-

\section{KEY POINTS}

- Urgent recanalization of the occluded cerebral artery in ischemic stroke is strongly associated with improved clinical outcomes.

- Mechanical thrombectomy using single-stent retrieval is effective in recanalizing large-vessel occlusion; however, in cases with refractory clots, dual stent retrievers can be used successfully as a rescue technique.

- This innovative off-label technique should be used with caution only in specific situations until larger prospective studies can determine its efficacy and safety for routine practice.

expanding stent. ${ }^{3,5-7}$ The unique design of the stent serves two functions: first, it acts as a temporary bypass by permitting immediate restoration of flow through the clot by expanding the stent within the clot, and second, it traps the clot within its stent struts, facilitating clot retrieval.

Currently, the most widely used stent retrievers are the Solitaire FR revascularization device (EV3 Neurovascular, Irvine, California) and Trevo Provue Retrieval System (Stryker Neurovascular, Kalamazoo, Michigan). Health Canada approved the Solitaire FR device in 2012 and the Trevo Provue system in 2013 for use in acute ischemic stroke. Both are equally effective, with higher recanalization rates and improved clinical outcomes compared with use of intravenous tissue plasminogen activator alone. ${ }^{3-7}$

\section{How is it delivered?}

Depending on the clinical status of the patient, this technique may be performed under either local or general anesthesia. With the use of an 8F balloon guide catheter (Merci; Concentric Medical, Mountain View, Calif.; or Cello; Covidien, Minneapolis, Minnesota) for the internal carotid artery or a 6 F Envoy DA guide catheter (Codman Neuro, Raynham, Massachusetts) for the basilar trunk, two of the same type of stent retriever (either Trevo or Solitaire FR; size $4 \times 20 \mathrm{~mm}^{2}$ ) are inserted in parallel or in a $Y$ configuration, with both tips ending in separate bifurcation 
limbs, for five minutes. Subsequently, both stents are gradually retrieved out of the guiding catheter, while continuous suction is maintained through the side port of the guide catheter. ${ }^{9,10}$ The decision to use this technique relies on several factors such as clot burden, local anatomy, duration and number of passages by a single stent retriever. ${ }^{10}$

Two examples of mechanical thrombectomy using dual stent retrievers are depicted in Figures 1 and 2. In these cases, both stents were deployed in kissing $Y$ configuration for five minutes and then retrieved simultaneously through the guiding catheter under continuous aspiration. A schematic diagram showing the technique is provided in Figure 3.

\section{Who is eligible?}

The inclusion and exclusion criteria for mechanical thrombectomy using the dual stent retriever technique are similar to standard single-stent retrieval. ${ }^{3-7,11}$ Eligible patients are adults 18 years of age or older (no upper age limit) with acute ischemic stroke caused by thromboembolic occlusion of a proximal intracranial artery in either the anterior or posterior circulation (e.g., internal carotid artery terminus, middle cerebral artery [M1 or M2 segment] and basilar trunk).
Intra-arterial thrombectomy should be started within six hours of symptom onset for anterior circulation stroke and 24 hours for posterior circulation stroke. Patients are selected if they score 2 or more on the National Institutes of Health Stroke Scale (range 0 to 42) and have a small infarct core on computed tomography (CT) or magnetic resonance imaging (an Alberta Stroke Program Early Computed Tomography Score [ASPECTS] greater than 6 in anterior circulation and absence of bilateral diffuse pontine ischemia in posterior circulation stroke). ${ }^{5,11}$

In addition to the previously mentioned criteria, the dual stent retrievers can be used specifically as a rescue technique for refractory thromboembolus at the carotid terminus, basilar tip and middle cerebral artery bifurcations, extending to both divisions, after single-stent retrieval failed., ${ }^{9,10}$

The exclusion criteria are large infarct core on baseline CT (ASPECTS of six or less, intracranial hemorrhage, severity of comorbid factors that may render the procedure unlikely to benefit, pregnancy and contraindication to iodinated contrast. ${ }^{3,5-7}$

\section{What are the harms?}

Incidence of complications using standard mechanical thrombectomy with single-stent retrieval is low. Reported complications

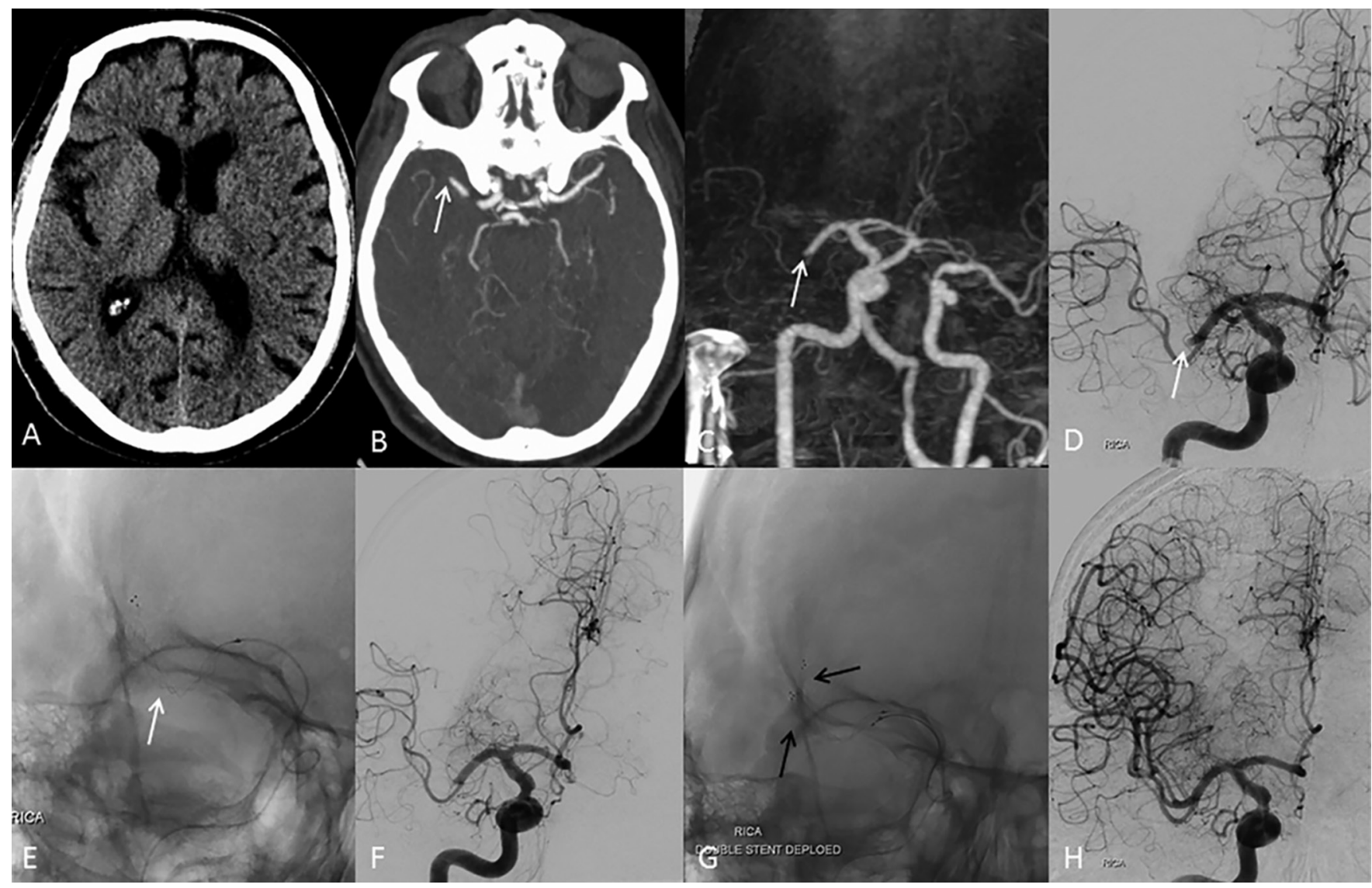

Figure 1: (A) Axial computed tomography (CT) scan of the head showing no obvious infarct. (B) Axial CT angiography and (C) coronal subtraction CT angiography of the head showing occlusion (white arrow) of the right middle cerebral artery (MCA). (D) A frontal angiogram showing an occluded right MCA. (E) Fluoroscopic anteroposterior image showing the deployed stent retriever (white arrow) in the right MCA. (F) Persistent occlusion of the right MCA after four passes with the stent retriever. (G) Fluoroscopic anteroposterior projection showing two stent retrievers deployed in the right MCA (black arrows). (H) Frontal angiogram showing complete recanalization of the right MCA. 


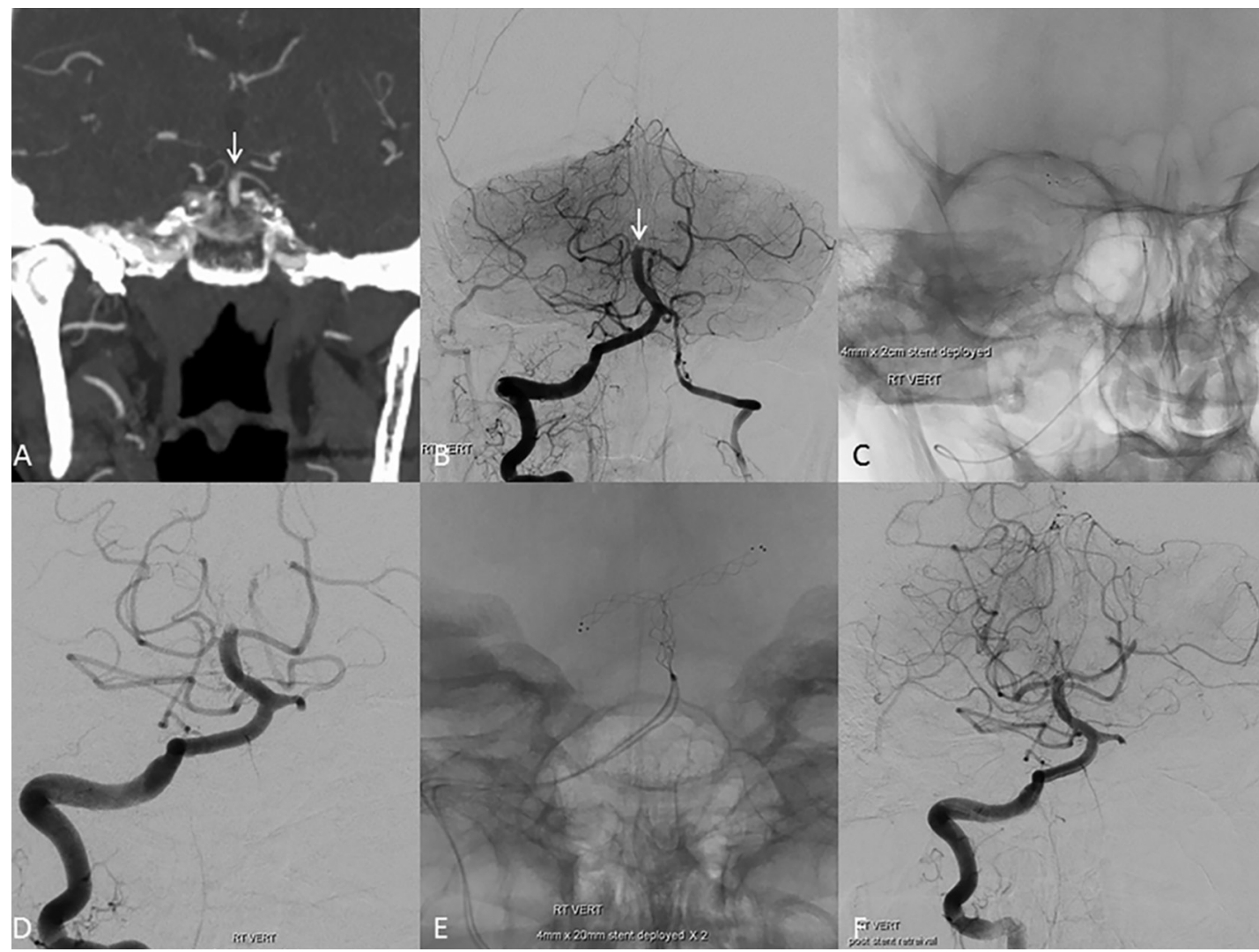

Figure 2: (A) Coronal and (B) frontal computed tomography (CT) angiograms of the head showing an occluded basilar tip (white arrow). (C) Fluoroscopic anteroposterior image showing the stent retriever deployed at the basilar tip. (D) Persistent occlusion of the basilar tip after three passes with a single stent retriever. (E) Fluoroscopic image showing two stent retrievers overlapped at the basilar trunk and their distal ends extended into the right and left posterior cerebral arteries (PCAs) to create the $\mathrm{Y}$ configuration. (F) Angiogram after dual-stent retrieval showing complete recanalization of the basilar tip with a residual clot in the distal left PCA.

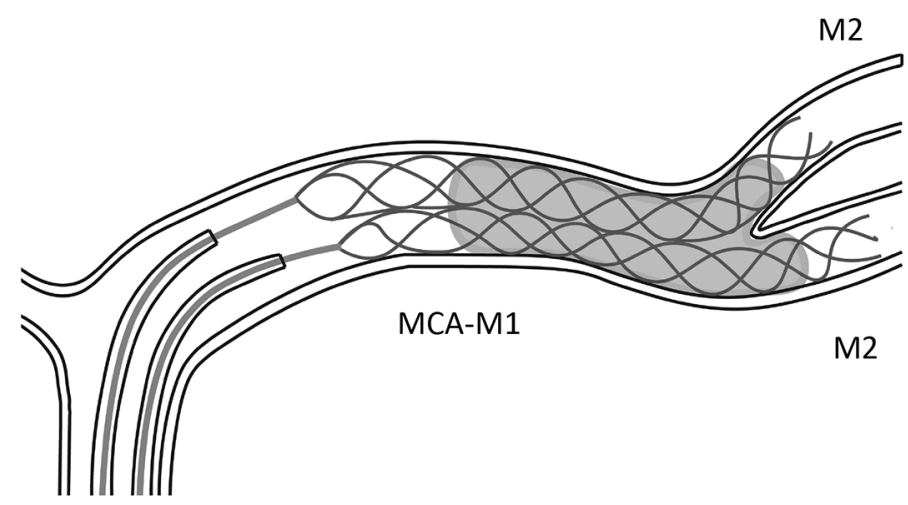

A

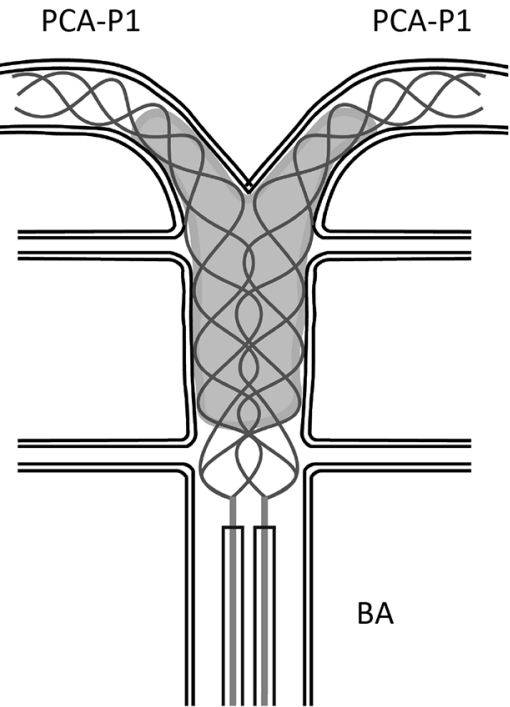

Figure 3: (A) Schematic diagram of the simultaneous deployment of dual stent retrievers within the clot in the middle cerebral artery (MCA) M1 (MCA-M1) segment with their distal ends extending to both limbs of the MCA bifurcation (M2 segments). (B) Schematic diagram showing the overlap of the two stent retrievers at the basilar trunk and their distal ends extending into both posterior cerebral arteries (PCA-P1 segments) to create the Y configuration. BA = basilar artery. 
include embolization into new territories outside the target vessel territory in $8.6 \%$, vessel dissections in $1.7 \%$ and vessel perforation in $0.9 \%$ of cases. ${ }^{3}$ However, the application of dual stent retrievers may further increase the risk of vessel injury by increasing exposure of more metals to the vessel endothelium during retrieval manoeuvres. The limited literature on this technique suggests that it is well tolerated with a comparable risk profile. ${ }^{9,10,12}$

Klisch and colleagues described one case of vessel perforation and seven cases of mild vasospasm with mechanical thrombectomy using dual stent retrievers as a rescue technique in 10 patients. There were no reported cases of severe vasospasm or arterial dissection. However, there were two deaths: one patient with malignant infarction following failed recanalization and the other with presumed reinfarction three weeks after initial successful recanalization. ${ }^{10} \mathrm{~A}$ single case report by Asadi and colleagues showed successful recanalization and no peri- or postprocedural complications with use of the dual stent retriever technique. ${ }^{9}$

\section{What is the evidence so far?}

There is limited evidence to date: only three articles have been published that report on the efficacy of dual stent retriever technique as a rescue treatment..$^{9,10,12}$ However, the results are promising, with comparable recanalization time and adverse events. According to Klisch and colleagues, this technique had an increased median total recanalization time of 60 minutes (interquartile range 45 to 87 minutes) compared with standard mechanical thrombectomy using single-stent retrieval (range 20 to 48 minutes). Complete recanalization (Thrombolysis in Cerebral Infarction grade of $2 \mathrm{~b} / 3$ ) was achieved in 8 out of 10 cases, with good clinical outcomes (modified Rankin Scale score of 2 or less) in five patients. ${ }^{10}$ Two other single-case reports also showed complete recanalization without peri- or postprocedural complications for retrieval of refractory clots in the anterior circulation after standard mechanical thrombectomy using singlestent retrieval failed. ${ }^{9,12}$

\section{What can be expected in the future?}

The available data and current limited experience have shown that dual stent receiver thrombectomy can be effective as a rescue technique for large-artery occlusion refractory to standard thrombectomy using a single stent retriever. Nevertheless, larger prospective studies are required to validate the usefulness and safety of this strategy and its ability to improve clinical outcomes. Until then, this technique should be used with caution in specific situations after giving adequate consideration to potential risks.

\section{References}

1. Rha JH, Saver JL. The impact of recanalization on ischemic stroke outcome: a meta-analysis. Stroke 2007;38:967-73.

2. Emberson J, Lees KR, Lyden P, et al. Effect of treatment delay, age, and stroke severity on the effects of intravenous thrombolysis with alteplase for acute ischaemic stroke: a meta-analysis of individual patient data from randomised trials. Lancet 2014;384:1929-35.

3. Berkhemer OA, Fransen PS, Beumer D, et al. A randomized trial of intraarterial treatment for acute ischemic stroke. N Engl J Med 2015;372:11-20.

4. Campbell BC, Mitchell PJ, Kleinig TJ, et al. Endovascular therapy for ischemic stroke with perfusion-imaging selection. N Engl J Med 2015;372:1009-18.

5. Goyal M, Demchuk AM, Menon BK, et al. Randomized assessment of rapid endovascular treatment of ischemic stroke. N Engl J Med 2015;372:1019-30.

6. Jovin TG, Chamorro A, Cobo E, et al. Thrombectomy within 8 hours after symptom onset in ischemic stroke. N Engl J Med 2015;372:2296-306.

7. Saver JL, Goyal M, Bonafe A, et al. Stent-retriever thrombectomy after intravenous t-PA vs. t-PA alone in stroke. N Engl J Med 2015;372:2285-95.

8. Gascou G, Lobotesis K, Machi P, et al. Stent retrievers in acute ischemic stroke: complications and failures during the perioperative period. AJNR Am J Neuroradiol 2014;35:734-40.

9. Asadi H, Brennan P, Martin A, et al. Double stent retriever technique in endovascular treatment of middle cerebral artery saddle embolus. J Stroke Cerebrovasc Dis. 2016;25:e9-e11.

10. Klisch J, Sychra V, Strasilla C, et al. Double Solitaire mechanical thrombectomy in acute stroke: Effective rescue strategy for refractory artery occlusions? AJNR Am J Neuroradiol 2015;36:552-6.

11. Gory B, Eldesouky I, Sivan-Hoffmann R, et al. Outcomes of stent retriever thrombectomy in basilar artery occlusion: an observational study and systematic review. J Neurol Neurosurg Psychiatry 2016;87:520-5.

12. Hsieh K, Verma RK, Schroth G, et al. Multimodal 3 Tesla MRI confirms intact arterial wall in acute stroke patients after stent-retriever thrombectomy. Stroke 2014; 45:3430-2.

\section{Competing interests: None declared.}

This article has been peer reviewed.

Affiliations: Department of Radiology (lancu), University of Ottawa; Department of Medical Imaging (Patro), The Ottawa Hospital, Ottawa, Ont.
Contributors: Both of the authors made substantial contributions to the design of the article, drafted the work, revised it critically for intellectual content, approved the final version to be published and agreed to be accountable for all aspects of the work.
Acknowledgements: The authors thank Dr. Prasad Hanagandi for assistance in editing the manuscript and Dr. Robert Moreland for preparing the schematic diagram of the dual stent retriever technique.

Correspondence to: Daniela lancu, diancu@ toh.on.ca 